\title{
Loss of Jak2 Impairs Endothelial Function by Attenuating Raf-1/MEK1/Sp-1 Signaling Along with Altered eNOS Activities
}

\author{
Ping Yang, ${ }^{* \dagger}$ Yawen Zhang, ${ }^{\ddagger \S}$ Junfeng Pang, ${ }^{\dagger}$ Shu Zhang, ${ }^{* \dagger}$ Qilin $\mathrm{Yu},{ }^{* \dagger}$ Long He, ${ }^{* \dagger}$ Kay-Uwe Wagner, ${ }^{\llbracket}$ Zhiguang Zhou, ${ }^{\| * *}$ and \\ Cong-Yi Wang*ł‡
}

\begin{abstract}
From the Center for Biomedical Research* and the Key Laboratory of Organ Transplantation, ${ }^{\dagger}$ Tongji Hospital, Tongji Medical College, Huazhong University of Science and Technology, Wuhan, China; the Center for Biotechnology and Genomic Medicine, ${ }^{\ddagger}$ Department of Pathology, Georgia Health Sciences University, Augusta, Georgia; the Department of Medicine, ${ }^{\S}$ Metabolic Disease Hospital, Tianjin Medical University, Tianjin, China; the Eppley Institute for Research in Cancer and Allied Diseases, ${ }^{\top}$ University of Nebraska Medical Center, Omaha, Nebraska; and the Key Laboratory of Diabetes Immunology, ${ }^{* *}$ Ministry of Education, and the Diabetes Center," Institute of Metabolism and Endocrinology, Second Xiangya Hospital, Central South University, Changsha, China
\end{abstract}

\author{
Accepted for publication \\ April 8, 2013 \\ Address correspondence to \\ Cong-Yi Wang, M.D., Ph.D., the \\ Center for Biomedical Research, \\ Tongji Hospital, Tongji Medical \\ College, Huazhong University of \\ Science and Technology, 1095 \\ Jiefang, Ave. Wuhan, 430030, \\ China; or Zhiguang Zhou, M.D. \\ Ph.D., Diabetes Center, Second \\ Xiangya Hospital, and Key \\ Laboratory of Diabetes Immu- \\ nology, Central South University, \\ Changsha, Hunan 410011, \\ China. E-mail: cwang@gru.edu \\ or zhouzg@hotmail.com.
}

\begin{abstract}
A number of inhibitors have been used to dissect the functional relevance of Jak2 in endothelial homeostasis, with disparate results. Given that Jak2 deficiency leads to embryonic lethality, the exact role of Jak2 in the regulation of postnatal endothelial function is yet to be fully elucidated. We generated a model in which Jak2 deficiency can be induced by tamoxifen in adult mice. Loss of Jak2 significantly impaired endothelium-dependent response capacity for vasodilators. Matrigel plug assays indicated a notable decrease in endothelial angiogenic function in Jak2-deficient mice. Studies in a hindlimb ischemic model indicated that Jak2 activity is likely to be a prerequisite for prompt perfusion recovery, based on the concordance of temporal changes in Jak2 expression during the course of ischemic injury and perfusion recovery. A remarkable delay in perfusion recovery, along with reduced capillary and arteriole formation, was observed in Jak2-deficient mice. Antibody array studies indicated that loss of Jak2 led to repressed eNOS expression. In mechanistic studies, Jak2 deficiency attenuated Raf-1/MEK1 signaling, which then reduced activity of Sp-1, an essential transcription factor responsible for eNOS expression. These data are important not only for understanding the exact role that Jak2 plays in endothelial homeostasis, but also for assessing Jak2-based therapeutic strategies in a variety of clinical settings. (Am J Pathol 2013, 183: 617-625; http://dx.doi.org/10.1016/j.ajpath.2013.04.007)
\end{abstract}

Endothelial cells (ECs) constitute the inner cellular lining of blood vessels and contribute importantly to vascular homeostasis in many pathophysiological settings, including vascular smooth muscle cell (VSMC) growth, vascular tone balance, and chronic inflammation. ${ }^{1}$ Moreover, appropriate endothelial function and angiogenesis are also critical in maintaining homeostasis of the body. For example, abnormal angiogenesis plays an essential role in tumor formation, progression, and metastasis, ${ }^{2-4}$ and suppression of angiogenesis is considered an effective approach for cancer therapy. ${ }^{5-7}$ In contrast, endothelial dysfunction and undermined vascular angiogenesis predispose to development of coronary artery disease, hypertension, stroke, and impaired post-traumatic recovery. ${ }^{8,9}$ Given that endothelial dysfunction is a common feature relevant to diverse cardiovascular disorders, ECs have received attention as a target or potential target to reverse these pathological processes. $^{10,11}$

Janus kinase 2 (Jak2) is one of four nonreceptor tyrosine kinases identified in mammals. ${ }^{12-14}$ It is a critical component of signal pathways implicated in cell growth, survival, proliferation, differentiation, and apoptosis. ${ }^{15,16}$ In particular,

Supported by the National Natural Science Foundation of China (81130014), the Chinese Ministry of Science \& Technology (2012BAI39B05), the European Foundation for the Study of Diabetes (EFSD)/Chinese Diabetes Society (CDS)/Lilly Program for Collaborative Diabetes Research between China and Europe, and the Synergy Award from the Diabetes Obesity Discovery Institute (DODI) at the Georgia Regents University (all to C.Y.W.).

P.Y. and Y.Z. contributed equally to this work. 
constitutive Jak2 expression has been detected in ECs, and Jak2 is thus implicated in homeostatic regulation of endothelial function. Indeed, previous studies revealed that abnormal Jak2 activation caused by hyperglycemia is detrimental to endothelial function, ${ }^{17}$ and Jak2 is indispensable for angiotensin II-induced hypertension. ${ }^{18,19}$ Although these discoveries are important, details of the underlying mechanism are largely unclear. Jak2 deficiency leads to embryonic lethality, but the exact role of Jak2 in regulation of postnatal endothelial function is yet to be fully elucidated. To address this issue, we generated an inducible Jak2 knockout mouse model in a B6 background by crossing Jak $2^{f l / f}$ mice with CreERT2 transgenic mice, in which Jak2 deficiency can be induced in adult mice. Loss of Jak2 significantly impaired endothelial response capacity for vasodilators. Interestingly, a decrease in endothelial angiogenic function was observed and, as a result, mice deficient in Jak2 exhibited a significant delay in perfusion recovery after hindlimb ischemic injury, compared with control mice. These findings contribute toward a fuller understanding of the exact role of Jak2 in regulation of endothelial homeostasis, and could also provide additional information for assessing Jak2-based therapeutic strategies in different clinical settings.

\section{Materials and Methods}

\section{Animals}

$J a k 2^{f l f l}$ mice $(H-2 b)$ have been described previously. ${ }^{20}$ These mice were backcrossed with Cre-ERT2 transgenic mice under the control of human ubiquitin $\mathrm{C}$ promoter to generate $\mathrm{Cre}^{+}-J a k 2^{f / f l}$ mice. ${ }^{21}$ The mice were housed in the specific pathogen-free animal facility of Georgia Regents University in microisolator cages supplied with autoclaved food and acidified water with a 12/12-hour light/dark cycle. All animal procedures were performed in accordance with the NIH Guide for the Care and Use of Laboratory Animals, 8th edition (2011) and were approved by the Georgia Regents University Institutional Animal Care and Use Committee.

\section{Induction of Jak2 Deficiency}

Male $\mathrm{Cr}^{+/+}-\mathrm{Jak} 2^{f / f l}$ mice were intraperitoneally injected with $25 \mathrm{mg} / \mathrm{kg}$ tamoxifen for five consecutive days at 8 weeks of age. Tamoxifen was freshly dissolved in corn oil before injection. $\mathrm{Cr}^{+/+}-\mathrm{Jak}^{f / f f}$ littermates injected with an equal volume of corn oil alone served as controls. Mice were sacrificed at 2 days after the last injection.

\section{Vascular Isometric Tension Assay}

After euthanasia, the thoracic aorta was removed and the surrounding fat tissue was cleaned in Krebs solution (115 $\mathrm{mmol} / \mathrm{L} \mathrm{NaCl}, 4.6 \mathrm{mmol} / \mathrm{L} \mathrm{KCl}, 1.2 \mathrm{mmol} / \mathrm{L} \mathrm{KH}_{2} \mathrm{PO}_{4}, 1.2$ $\mathrm{mmol} / \mathrm{L} \mathrm{MgSO}_{4}, 2.5 \mathrm{mmol} / \mathrm{L} \mathrm{CaCl}_{2}, 25 \mathrm{mmol} / \mathrm{L} \mathrm{NaHCO}_{3}$, $11.1 \mathrm{mmol} / \mathrm{L}$ glucose, and $0.02 \mathrm{mmol} / \mathrm{L}$ EDTA; $\mathrm{pH}$ 7.4). Aorta segments (approximately $2 \mathrm{~mm}$ long) were mounted onto a four-channel tissue bath myograph system (Catamount Research and Development, St. Albans, VT) for isometric tension recording after equilibrating in Krebs solution for approximately 1 hour and were gassed with $5 \% \mathrm{CO}_{2}$ and $95 \%$ $\mathrm{O}_{2}$. To test relaxation of arteries precontracted with $10^{-5} \mathrm{~mol} / \mathrm{L}$ phenylephrine, $120 \mathrm{mmol} / \mathrm{L} \mathrm{KCl}$ and $10^{-9}$ to $10^{-5} \mathrm{~mol} / \mathrm{L}$ phenylephrine were used as vasoconstrictors and $10^{-9}$ to $10^{-5}$ $\mathrm{mol} / \mathrm{L}$ acetylcholine and $10^{-9}$ to $10^{-5} \mathrm{~mol} / \mathrm{L}$ sodium nitroprusside were used as vasodilators; $10^{-4} \mathrm{~mol} / \mathrm{L}$ of the L-arginine competitor L-nitroarginine-methylester (L-NAME) was used to inhibit endogenous endothelial nitric oxide synthase (eNOS) activity. Dose-response curves were generated cumulatively.

\section{Matrigel Plug Assay}

Jak2-deficient and control mice were injected subcutaneously on both sides of the back, each side with $0.5 \mathrm{~mL}$ icecold 1:2 diluted Matrigel (BD Biosciences, San Jose, CA) containing $200 \mathrm{ng} / \mathrm{mL}$ vascular endothelial growth factor (VEGF) (R\&D Systems, Minneapolis, MN) and $60 \mathrm{U} / \mathrm{mL}$ heparin (Sigma-Aldrich, St. Louis, MO). One week later, the mice were sacrificed using $\mathrm{CO}_{2}$ and the gel plugs were harvested. Part of the plugs was subjected to immunostaining of CD31 expression; the remainder was weighed, minced into small pieces, and immersed in $500 \mu \mathrm{L}$ distilled water at $4{ }^{\circ} \mathrm{C}$ overnight. The amount of hemoglobin in the plugs was determined using Drabkin reagent (SigmaAldrich) according to the manufacturer's instruction.

\section{Immunostaining of Plug or Tissue Sections}

Sections $(10 \mu \mathrm{m}$ thick) prepared from Matrigel plugs or ischemic tissues were used for immunostaining with rat antimouse CD31 antibody (BD Pharmingen, San Diego, CA) to detect endothelial cells, followed by staining with a Texas Red-conjugated goat anti-rat IgG (Santa Cruz Biotechnology, Santa Cruz, CA). Nuclei were stained blue with 0.2 $\mu \mathrm{g} / \mathrm{mL}$ DAPI. Four mice were analyzed for each study group.

\section{Hindlimb Ischemic Model and Assessments}

Unilateral hindlimb surgery was performed as reported previously. ${ }^{22}$ Hindlimb blood flow in mice subjected to hindlimb ischemia was measured with a PIM 3 scanning laser Doppler blood perfusion imaging system (Perimed, Stockholm, Sweden). Animals were anesthetized and maintained at $37^{\circ} \mathrm{C}$ on a heating plate, to minimize temperature variation. Laser Doppler blood flow analyses were performed on legs and feet before surgery and on days $0,2,4,12$, and 16 after surgery. Blood flow images of hindlimbs were then acquired (three images per animal). Blood flow data were expressed as change in laser frequency, represented by pixel color. Mean hindlimb blood flow was calculated as the ratio of blood flow on the ischemic side to blood flow on the nonischemic side. Six mice were analyzed in each group. 
Antibody Array, Immunoprecipitation, and Western Blot Analysis

Antibody array detection was performed using a kit from Spring Bioscience (Pleasanton, CA) according to the manufacturer's instructions. Aorta lysates (100 $\mu \mathrm{g}$ protein) derived from wild-type or $J a k 2^{-/-}$mice were labeled with biotin using an antibody array detection kit (AMD-001) and then conjugated to the master antibody array (AMS-700), both from Spring Bioscience. Cy3-streptavidin was used to detect the bound proteins. Signals were scanned with a Typhoon 9400 scanner and data were collected and analyzed using the associated ImageQuant software version 7.0 (GE Healthcare Biosciences, Piscataway, NJ). Differentially expressed proteins were then confirmed by Western blotting as described previously. ${ }^{23}$ For immunoprecipitation, an Sp-1 antibody $(5 \mu \mathrm{g} / \mathrm{mL}$; Upstate; EMD Millipore, Billerica, MA) was added into the precleared lysates for 1 hour at $4^{\circ} \mathrm{C}$, followed by incubation with $50 \mu \mathrm{L} / \mathrm{mL}$ protein $\mathrm{A} / \mathrm{G}$ Plus agarose beads (Sigma-Aldrich) overnight at $4^{\circ} \mathrm{C}$. The resulting precipitates were washed five times with $1 \mathrm{~mL}$ of ice-cold PBS and then were eluted from the agarose beads by boiling for 5 minutes in SDS sample buffer. The resulting products were then analyzed by Western blotting. The Jak2 antibody was obtained from EMD Millipore (Billerica, MA), and antibodies for MEK1, p-MEK1, AKT, p-AKT, ERK1/2, pERK1/2; p38 and p-p38 were obtained from Cell Signaling Technology (Danvers, MA); antibodies against total and phosphorylated eNOS and Raf-1 proteins and against $\alpha$-tubulin were obtained from Santa Cruz Biotechnology.

\section{Electrophoretic Mobility Shift Assay}

Nuclear proteins were extracted from porcine aortic endothelial cells (PAECs) after treatment with $10 \mathrm{U} / \mathrm{mL}$ tumor necrosis factor $\alpha$ (TNF- $\alpha$ ) (Roche Diagnostics, Indianapolis, IN), along with $50 \mu \mathrm{mol} / \mathrm{L}$ of the MEK1 inhibitor PD98059 (Calbiochem, San Diego, CA) or control vehicle for 8 hours or treatment with $0.5 \mu \mathrm{mol} / \mathrm{L}$ of the Jak2 inhibitor AZD1480 (ChemieTek, Indianapolis, IN) for 12 hours using a NE-PER nuclear and cytoplasmic extraction kit (Thermo Scientific, Pittsburgh, PA). Electrophoretic mobility shift assay (EMSA) was performed using a Pierce LightShift chemiluminescent EMSA kit (Thermo Fisher Scientific, Rockford, IL) as previously reported. ${ }^{22}$ A biotin-labeled eNOS promoter probe, $5^{\prime}$-ATTGTGTATGGGATAGGGGCGGGGCGAG-3' (GenScript, Piscataway, NJ) was used.

\section{Statistical Analysis}

Data are expressed as means \pm SEM. Constriction responses are expressed as the maximum response produced by the individual compounds. Concentration-response curves were fitted using a nonlinear interactive fitting program (GraphPad Prism software version 5.0; GraphPad Software, San Diego, $\mathrm{CA}$ ), and statistical analyses of the data were performed using Student's $t$-test. For blood flow imaging data, repeatedmeasures analysis of variance was used. A three-way repeated-measures analysis of variance was used to analyze the differences in blood flow between Jak2-deficient and control mice. $P<0.05$ was considered statistically significant.

\section{Results}

\section{Loss of Jak2 Impairs Endothelium-Dependent Response to Vasodilators}

$\mathrm{Cr} e^{+/+}-\mathrm{Jak} 2^{f / f l}$ mice were generated by breeding $\mathrm{Jak} 2^{f / f l}$ mice with Cre-ERT2 transgenic mice as described above. To induce Jak2 deficiency, 8-week-old male $\mathrm{Cr}^{+/+}{ }_{-J a k} 2^{f / f l}$ mice were intraperitoneally injected with $25 \mathrm{mg} / \mathrm{kg}$ tamoxifen for five consecutive days. Male littermates administered with only the corn-oil carrier solution were used as controls. To confirm Jak2 deficiency, the mice were sacrificed 2 days after the last injection, and aorta lysates were prepared for Western blot analysis. High levels of Jak2 were detected in corn oil-injected mice, whereas Jak2 was absent in tamoxifen-induced mice (Figure 1A), indicating that tamoxifen efficiently induced Jak2 deficiency in $\mathrm{Cr}^{+/+}-\mathrm{Jak} 2^{f l / f}$ mice. To further confirm that Jak2 down-regulation after tamoxifen induction is equal and complete in all vascular cell types (in the present study, ECs and VSMCs), we performed immunostaining of aortic sections. Steady levels of Jak2 expression were detected in ECs and VSMCs of mice injected with corn oil alone (controls) (Figure 1B), whereas Jak2 was nearly absent in ECs and VSMCs of aortic sections derived from tamoxifen-induced mice (Figure 1C).

To address the effect of Jak2 deficiency on endothelial function, we first examined endothelial response capacity for vasoconstrictors and vasodilators. To this end, the mice were sacrificed after day 2 of tamoxifen induction, and aorta segments were prepared for contractility assays as described above. No significant difference was observed between Jak2-deficient and control mice for contractility induced by either $120 \mathrm{mmol} / \mathrm{L} \mathrm{KCl}$ (Figure $2 \mathrm{~A}$ ) or $1 \times 10^{-10}$ to $1 \times 10^{-5} \mathrm{~mol} / \mathrm{L}$ phenylephrine (Figure 2B). However, Jak2-deficient mice exhibited significantly impaired relaxation in response to $1 \times 10^{-9}$ to $1 \times 10^{-5}$ $\mathrm{mol} / \mathrm{L}$ acetylcholine and, in contrast, a maximum relaxation was achieved in littermate controls (Figure 2C). Importantly, after blockade of endogenous endothelium-derived nitric oxide (NO) by administration of $1 \times 10^{-4} \mathrm{~mol} / \mathrm{L}$ L-NAME, both Jak2deficient and control mice exhibited similar capacity in response to $1 \times 10^{-10}$ to $1 \times 10^{-6} \mathrm{~mol} / \mathrm{L}$ sodium nitroprusside-induced relaxation (Figure 2D), indicating that the reduced relaxation capacity in Jak2-deficient mice was caused by the impaired endogenous NO production from ECs. Taken together, these data demonstrate that loss of Jak2 attenuates endothelium-dependent response capacity for vasodilators.

\section{Jak2 Is Essential for Endothelial Angiogenesis}

We next addressed the effect of Jak2 deficiency on endothelial angiogenesis, using a Matrigel plug assay. At day 2 

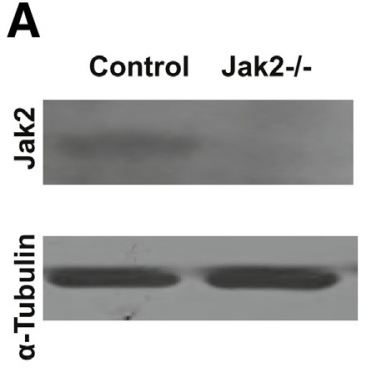

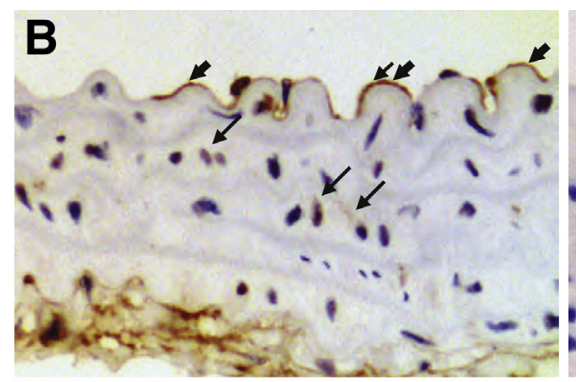

littermate control

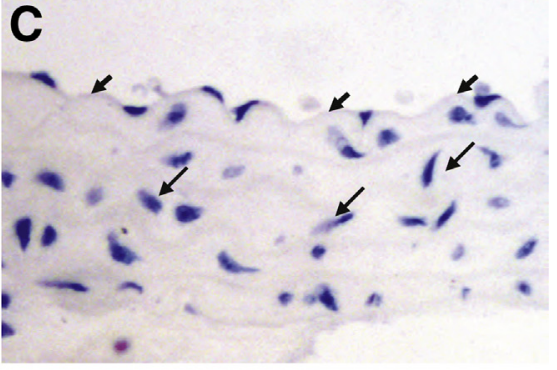

Jak2-/-

Figure 1 Tamoxifen efficiently induced Jak2 down-regulation in blood vessels. A: Western blot analysis demonstrates Jak2 deficiency in aortic lysates after tamoxifen induction in $\mathrm{Cre}^{+}-\mathrm{Jak} \mathrm{f}^{\mathrm{fl} f l}$ mice. B and C: Jak2 immunostaining of aortic section from representative control (B) and tamoxifen-induced (C) mice. Steady levels of Jak2 were detected in all control mice examined, but Jak2 was nearly absent in all tamoxifen-induced mice. Importantly, tamoxifen-induced Jak2 downregulation was equal and complete across vascular cell types. Selected ECs and VSMCs are indicated by arrows. Original magnification, $\times 400 . n=4$ mice per group.

after the last tamoxifen induction, the mice were anesthetized and subcutaneously injected with $0.5 \mathrm{~mL}$ ice-cold Matrigel (BD Biosciences) on each side of the back; the gel plugs were removed at 1 week after injection. A robust angiogenic response was seen in the Matrigel plugs derived from control mice, whereas a significantly lower amount of neovessels inside the Matrigel plugs was observed in Jak2deficient mice, as assessed by intact red blood cell content (Figure 3A). Hemoglobin assay confirmed that hemoglobin content in the gel plugs originated from littermate controls was 3.3-fold higher than that from Jak2-deficient mice $(0.99 \pm 0.23 \mu \mathrm{g} / \mathrm{mg}$ gel versus $0.23 \pm 0.1 \mu \mathrm{g} / \mathrm{mg}$ gel,
A
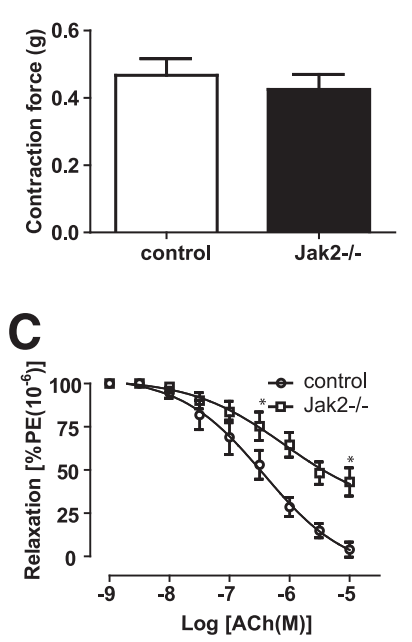

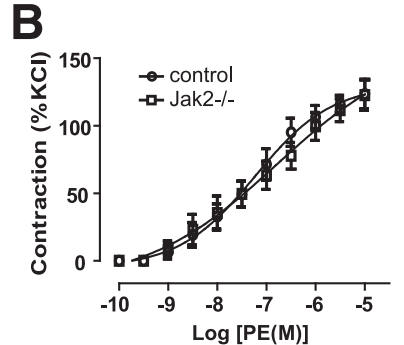

\section{D}

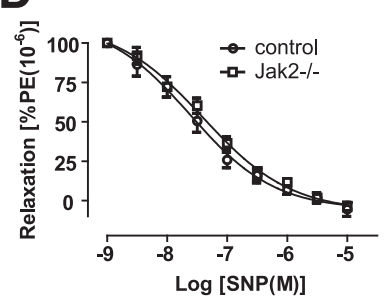

Figure 2 Loss of Jak2 impairs endothelial response capacity for vasodilators. Aortic rings were prepared from both Jak2-deficient and control mice and then were subjected to isometric contractility assay. A: Vascular constriction induced by $\mathrm{KCl}(120 \mathrm{mmol} / \mathrm{L})$. B: Phenylephrine-induced vascular contractility. C: Endothelium-dependent relaxation induced by acetylcholine. D: Endothelium-independent relaxation induced by sodium nitroprusside after blockade of endogenous eNOS by $10^{-4} \mathrm{~mol} / \mathrm{L}$ L-NAME. No difference for vascular contractile activity was observed between Jak2deficient and control mice, but Jak2-deficient mice exhibited a significantly impaired endothelium-dependent relaxation. Data are expressed as means \pm SEM. $n=4$ mice per group. ${ }^{*} P<0.01$. ACh, acetylcholine; M, mol/L; PE, phenylephrine; SNP, sodium nitroprusside. respectively; $P<0.001$ ) (Figure 3B). Immunostaining of the gel plugs revealed that control mice exhibited significantly greater numbers of $\mathrm{CD} 31^{+}$cells (ECs) and of tubular-like structures, compared with Jak2-deficient mice (Figure 3C). Taken together, these data support the notion that loss of Jak2 impairs endothelium-dependent angiogenesis.

\section{Jak2 Deficiency Attenuates Perfusion Recovery after Hindlimb Ischemic Injury}

Next, a hindlimb ischemia model was used to assess perfusion recovery, to further confirm the above ex vivo findings. We first examined Jak2 temporal changes in expression in control mice during the course of ischemic injury and perfusion recovery by Western blot analysis (Figure 4A) Interestingly, a significant reduction in Jak2 expression was observed on day 1 after ischemic surgery; however, a steady increase for Jak2 expression was observed from day 2 after the surgery, and the highest level was observed on day 7 . After day 7, Jak2 exhibited a steady decrease and returned to a level similar to the physiological condition at day 0 , before ischemic surgery (Figure 4B). This concordance of Jak2 upregulation along with ischemic injury and Jak2 downregulation along with perfusion recovery indicates that Jak2 may play a pivotal role in perfusion recovery after an ischemic attack.

To address this possibility, unilateral hindlimb ischemia was induced in both Jak2-deficient mice and littermate controls. All mice survived the surgical procedure, and blood flow in ischemic (left side) and nonischemic (right side) limbs was monitored using a laser Doppler imaging system as described above (Figure 5A). Blood flow in the ischemic hindlimbs was completely undetectable in both Jak2-deficient and control mice immediately after femoral artery excision. Interestingly, littermate controls exhibited possible evidence of blood flow restoration on day 4 after ischemic surgery, and distinctive restoration of blood flow $(30.8 \%)$ was detected on day 8 . On average, perfusion recovery reached approximately $53.8 \%$ on day 12 and was nearly complete on day $16(74.4 \%)$ in all control mice 
A
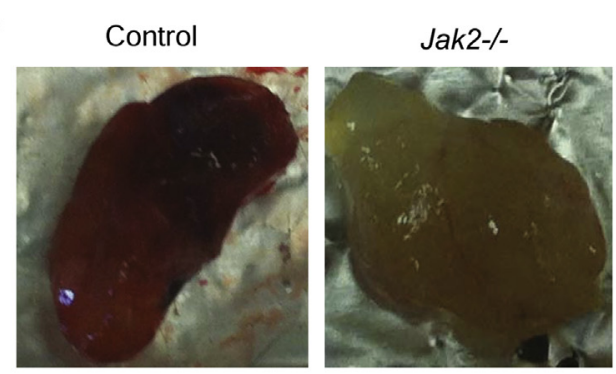

B

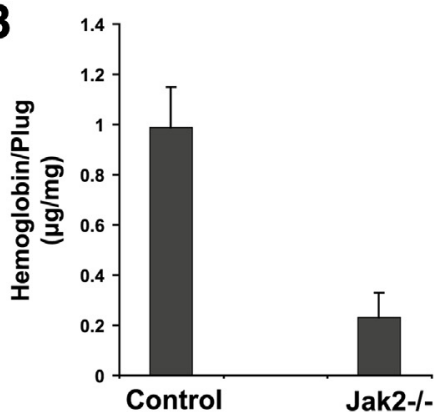

C
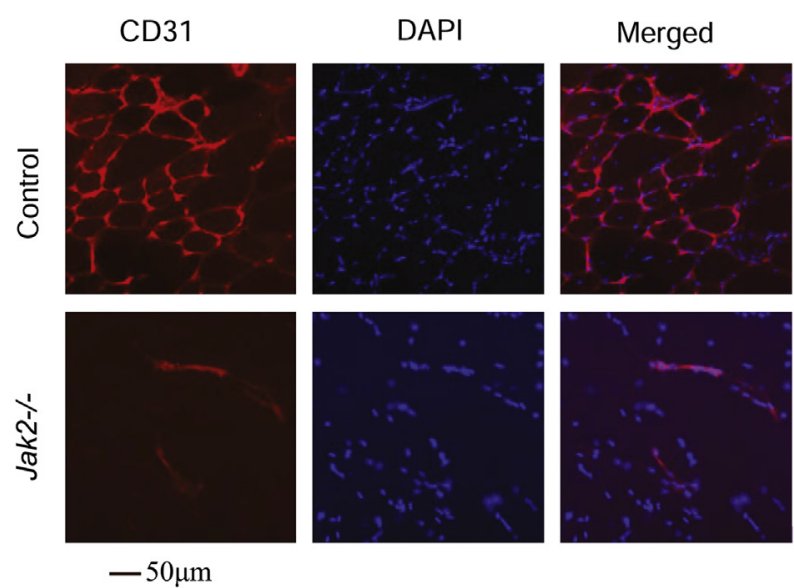

Figure 3 Mice deficient in Jak2 exhibit reduced capacity for endothelial angiogenesis, as demonstrated by Matrigel plug assays. A: Representative images of gel plugs. B: Hemoglobin content of gel plugs derived from Jak2-deficient and control mice. C: Representative microscopic images of sections from gel plugs. The sections were stained with an antibody against CD31 (a marker for ECs) as an indicator of vascular neogenesis, and DAPI was used to stain cell nuclei. Matrigel plugs were collected from mice at 1 week after injection. Data are expressed as means \pm SEM. $n=5$ mice per group. analyzed (Figure 5B). In sharp contrast, no detectable blood flow restoration was observed in Jak2-deficient mice until day $8(11.9 \%)$, and blood flow was restored to only approximately $29.2 \%$ and $39.7 \%$ at days 12 and 16 after ischemic surgery, respectively (Figure 5B).

To further confirm these findings, we determined the differences in neovascularization by examining capillary $\left(\mathrm{CD} 31^{+}\right.$cells $)$and arteriole $\left(\alpha\right.$-actin ${ }^{+}$cells) formation. The number of $\mathrm{CD}^{+} 1^{+}$cells (ECs) (Figure 5C) and $\alpha$-actin ${ }^{+}$ cells (smooth muscle cells) (Figure 5D) were significantly higher in the ischemic sections of control mice than in those of Jak2-deficient mice. Taken together, these data suggest that Jak2 deficiency impairs endothelium-dependent neovascularization and thereby delays blood flow perfusion recovery after an ischemic attack.

\section{Jak2 Deficiency Reduces eNOS Expression and Activity}

To dissect the underlying mechanisms by which Jak2 deficiency impairs endothelial function, we performed antibody array analysis using antibodies against signaling molecules essential for endothelial function. Remarkably, a significant reduction in eNOS expression was observed in Jak2deficient mice, compared with controls. There was also evidence suggesting potential reduction in AKT, p38, and ERK1/2 levels in Jak2-deficient mice. Western blot analysis of aortic lysates was used to confirm these data. Consistent with the antibody array results, total eNOS expression was significantly lower in Jak2-deficient mice than in controls. We then examined eNOS activity by analysis of the phosphorylated form of eNOS. As expected, moderate levels of p-eNOS was consistently detected in control mice, but p-eNOS was nearly undetectable in Jak2-deficient mice (Figure 6A). However, Western blot analysis failed to detect changes for both total and phosphorylated AKT (Figure 6B), ERK1/2 (Figure 6C), and p38 (data not shown).

To exclude the possibility that the reduced eNOS expression observed in $J a k 2^{-/-}$mice was actually a consequence of altered endothelial integrity, we further examined the differences in ECs by Western blot analysis of CD31 expression in aortic lysates. We failed to detect any difference in CD31 expression between Jak2 ${ }^{-/-}$and control mice (Figure 6D), suggesting that altered eNOS expression is probably directly caused by Jak2 deficiency. Overall, these results suggest that loss of Jak2 represses eNOS expression, and that this effect is likely independent of AKT, ERK1/2, and p38 signaling.
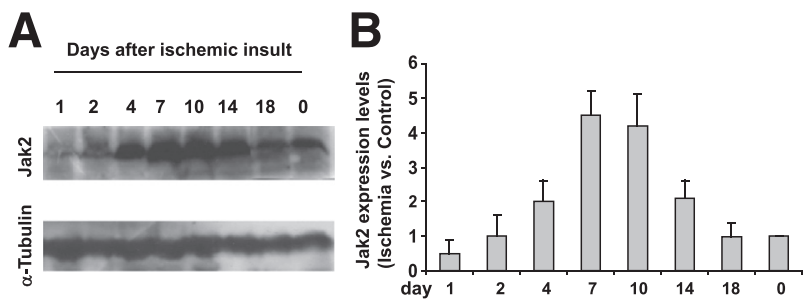

Figure 4 Jak2 undergoes a temporal change in expression after ischemic insult. A: Representative Western blots show temporal change in Jak2 expression after ischemic surgery. B: Relative Jak2 expression at each time point examined, normalized by $\alpha$-tubulin. Data are expressed as means \pm SEM. $n=5$ mice per group. 


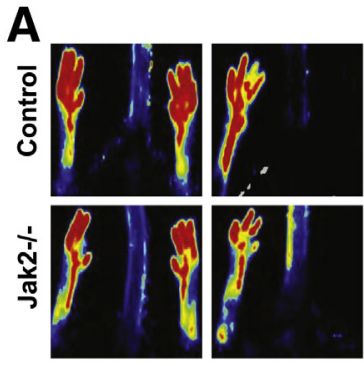

BS

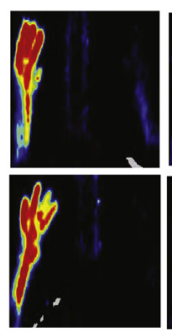

Day 4

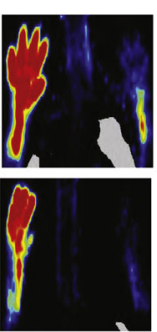

Day 8

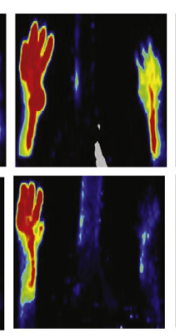

Day 12

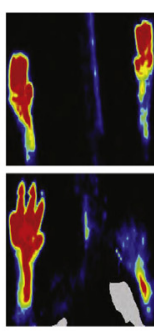

Day 16

BS: Before Surgery

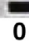

1000

C
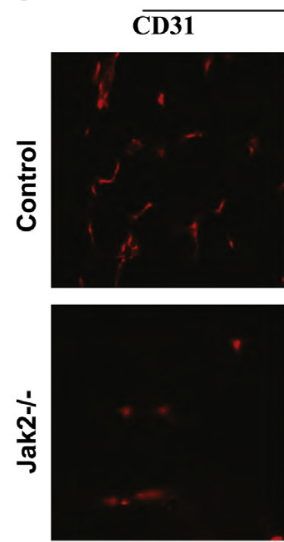

Endothelial Cells

DAPI merge

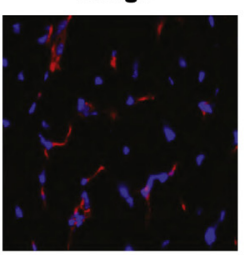

D

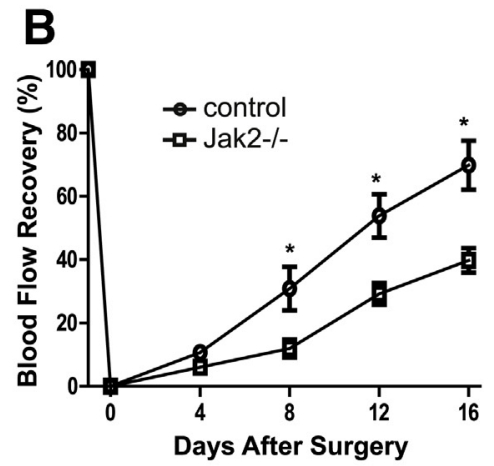

Smooth Muscle Cells
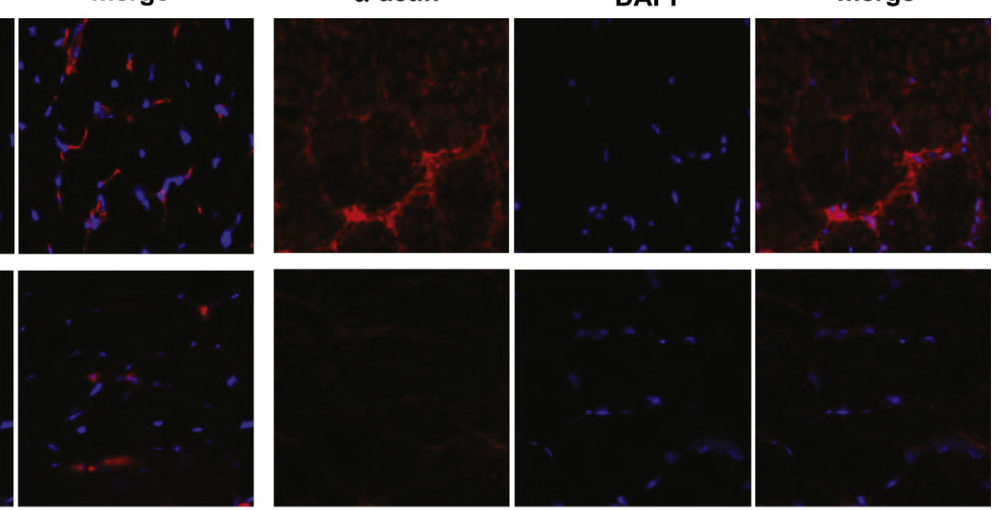

Figure 5 Jak2 deficiency attenuates blood flow perfusion recovery after hindlimb ischemic surgery. A: Representative images collected by a laser Doppler imaging system during the course of restoration of blood flow after hindlimb ischemic surgery. Blood flow was measured using a laser Doppler imaging system and is expressed as change in laser frequency (indicated by pixel color). B: Average blood flow at each time point for all mice examined. Mean hindlimb blood flow was calculated as the ratio of ischemic (left side) to nonischemic (right side) blood flow. C: Jak2-deficient mice exhibited significantly impaired capillary formation at day 7 of ischemic injury, compared with that of controls. The amount of ECs $\left(C D 31^{+}\right)$in hindlimb sections served as an indicator of capillary formation. D: Arteriole formation at day 7 of ischemic injury. The amount of smooth muscle cells $\left(\alpha-\right.$ actin $\left.^{+}\right)$in hindlimb sections served as an indicator of arteriole formation. $n=4$ mice per group. ${ }^{*} P<0.01$. Original magnification, $\times 200$ (C and D).

\section{Loss of Jak2 Attenuates Sp-1 Activity and Thereby Represses eNOS Expression}

These findings prompted us to examine the transcriptional activity of Sp-1, which is an essential transcription factor responsible for eNOS expression by binding to the GC box in the proximal eNOS promoter. Pooled aorta lysates originated from Jak2-deficient and control mice were subjected to immunoprecipitation with an $\mathrm{Sp}-1$ antibody as described above, and the resulting products were analyzed by Western blot analysis for total and serine/threonine phosphorylated Sp-1 (p-Sp-1). Notably, loss of Jak2 led to at least a twofold decrease for the activated form of Sp-1 (ie, $\mathrm{p}-\mathrm{Sp}-1$ ), whereas total Sp-1 levels remained unchanged (Figure 7A). To further confirm this finding, we treated PAECs with 0.50 $\mu \mathrm{mol} / \mathrm{L}$ AZD1480, a Jak2 inhibitor, and nuclear lysates were then prepared for EMSA to assess the capacity of Sp-1 for binding to the eNOS promoter. The AZD1480 treatment reduced Sp-1 DNA binding activity by nearly fourfold (Figure 7B). Taken together, these data suggest that Jak2 deficiency attenuates endothelial Sp-1 transcriptional activity, which then represses eNOS expression. ${ }^{24,25}$

\section{Jak2 Deficiency Impairs Raf-1/MEK1 Signaling and Thereby Suppresses Sp-1 Activity}

To dissect the mechanisms underlying Jak2 regulation of Sp-1 transcriptional activity, we analyzed all kinase signaling molecules downstream of Jak2 but upstream of Sp-1. Notably, loss of Jak2 significantly attenuated tyrosine phosphorylation (at 340 and 341) of Raf-1 (Figure 7C), a serine/threonine kinase that transmits signals essential for cell growth and proliferation. ${ }^{26}$ Consistent with this finding, activation of the direct downstream target $\mathrm{MEK}^{27}$ was significantly affected, as indicated by at least a twofold reduction in p-MEK1 in Jak2-deficient mice, compared with control mice (Figure 7D). Given the essential role of MEK1 in Sp-1 activation observed in hepatocytes, ${ }^{28}$ we assumed that Jak2 deficiency impairs Raf-1/MEK1 signaling, which then attenuates $\mathrm{Sp}-1$ activation and thereby represses eNOS expression in ECs. To test this assumption, we analyzed Sp1 transcriptional activity in PAECs after treatment with TNF- $\alpha$ along with the MEK1 inhibitor PD98059. As expected, inhibition of MEK1 activity significantly attenuated TNF- $\alpha$-induced Sp-1 phosphorylation (Figure 7E) and also 


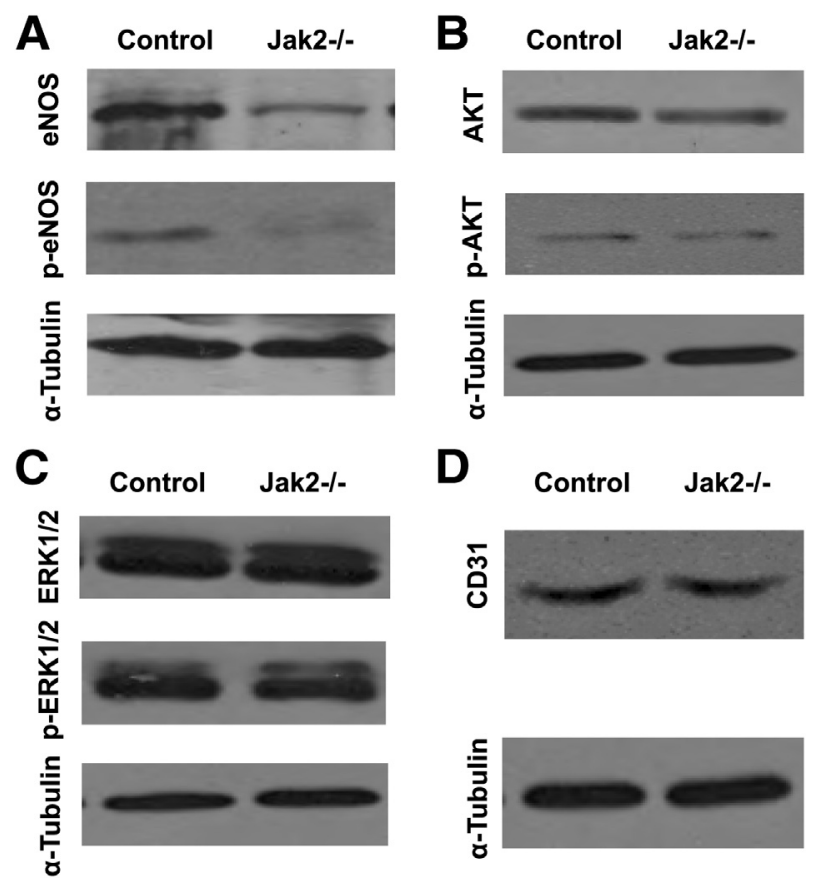

Figure 6 Jak2 deficiency leads to significant reduction in eNOS expression. Western blot analysis was used to confirm the data obtained from antibody array analysis using aortic lysates between corn oil-injected mice (Control) and tamoxifen-induced mice $\left(\mathrm{Jak2}^{-/-}\right)$. A: eNOS and p-eNOS expression. B: AKT and p-AKT expression. C: ERK1/2 and p-ERK1/2 expression. D: CD31 expression. Jak2 deficiency significantly impaired expression of both eNOS and p-eNOS, but it did not cause any distinctive change in expression of AKT or p-AKT, nor of ERK1/2 or p-ERK1/2. Of note, there was no perceptible difference for CD31 expression between control and $J a k 2^{-1-}$ mice, suggesting that the reduction of eNOS expression in Jak2 $2^{-1-}$ mice was not a consequence of altered endothelial integrity. Aortic lysates pooled from two mice were used for the analysis, and three pools were analyzed for each study group.

reduced DNA binding activity to the eNOS promoter (Figure 7F). Taken together, these data suggest that the reduced eNOS expression observed in Jak2-deficient mice was likely caused by the impaired Raf-1/MEK1 signaling and the resulting attenuated Sp-1 transcriptional activity.

\section{Discussion}

The Janus kinase family (JAKs) consists of four nonreceptor tyrosine kinases: JAK1, JAK2, JAK3, and TYK2. All of them share a similar structure composed of a kinase domain, a regulatory pseudo-kinase domain, an $\mathrm{SH} 2$ domain, and a FERM domain. Gene-targeted studies have demonstrated essential, nonredundant functions for all JAKs, and therefore, they have emerged as critical molecules in mammalian development, physiology, and disease. Mice deficient in Jak1 develop early postnatal lethality due to neurological deficits, whereas loss of Jak3 predisposes to the development of severe combined immunodeficiency, and Tyk2 deficiency is associated with parasite infection and resistance to lipopolysaccharide. ${ }^{29}$ In contrast, Jak2 deficiency is embryonic lethal, and therefore most Jak2-related studies have been conducted with chemical inhibitors. ${ }^{14}$ Thus, the exact role of Jak2, and particularly the role of Jak2 in regulation of postnatal endothelial function, is yet to be fully elucidated.

In the present study, we generated an inducible model in which Jak2 deficiency can be induced by tamoxifen in adult mice (Figure 1). Our focus was on the role of Jak2 in the pathogenesis of endothelial dysfunction, a condition associated with cardiovascular disorders in a variety of clinical settings. We first observed that mice deficient in Jak2 exhibited a significantly impaired response capacity for vasodilators such as acetylcholine (Figure 2C), although their response to vasoconstrictors remained the same (Figure 2, A and B), suggesting that Jak2 activity might be essential for the homeostasis of endothelial function. This observation seemingly conflicts with previous findings of enhanced Jak2 activity associated with endothelial dysfunction in the settings of diabetic complications ${ }^{17}$ and angiotensin
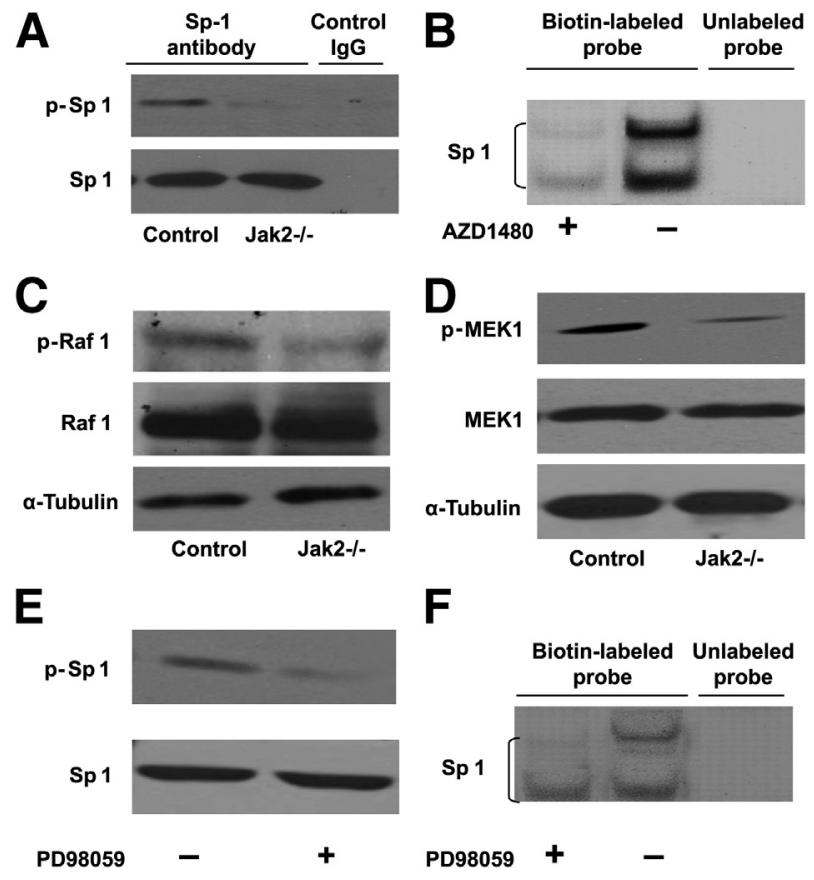

$\mathbf{F}$

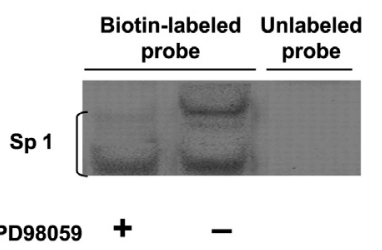

Figure 7 Loss of Jak2 attenuates the Raf-1/MEK1/Sp-1 signaling axis and thereby represses eNOS expression. A: Western blot analysis of immunoprecipitates revealed that loss of Jak2 repressed Sp-1 transcriptional activity, as indicated by reduced p-Sp-1 levels. B: Inhibition of Jak2 in PAECs resulted in nearly a fourfold reduction in Sp-1 binding to the eNOS promoter. PAECs were used to confirm the data for Jak2-deficient mice (A). PAECs were treated with either the Jak2 inhibitor AZD1480 or vehicle, and nuclear proteins were prepared and subjected to EMSA to assess activity for Sp-1 binding to eNOS promoter. C: Western blot analysis of Jak2 downstream kinases revealed impaired phosphorylation of tyrosines (Tyr340/ 341) for Raf-1 after loss of Jak2. D: Activation of MEK1, a serine-threonine protein kinase downstream of Raf-1, was significantly attenuated in Jak2deficient mice, as indicated by reduced levels of p-MEK1. E: Western blot analysis of TNF- $\alpha$-induced PAEC lysates along with the MEK1 inhibitor PD98059 or vehicle treatment. Inhibition of MEK1 activity significantly repressed TNF- $\alpha$-induced phosphorylation of Sp-1, as indicated by reduced p-Sp-1 levels. F: Inhibition of MEK1 activity resulted in a reduced capacity for Sp-1 binding to eNOS promoter. EMSA was used with nuclear proteins derived from TNF- $\alpha$-induced PAECs treated with MEK1 inhibitor or vehicle. 
II-induced hypertension. ${ }^{18,19}$ The discrepancy is likely due to the differences between physiological and pathological settings. We assume that well-tuned Jak2 activity is required for endothelial homeostasis, and that loss of Jak2 or sustained abnormal Jak2 activation (eg, under diabetic conditions) would predispose to the development of endothelial dysfunction. Another contributing factor to the discrepancy could be the use of chemical inhibitors, such as AG490, that nonspecifically target molecules other than Jak2. ${ }^{14}$ Nevertheless, these discoveries, together with the present data, support the notion that altered Jak2 activity is implicated in the pathogenesis of endothelial dysfunction.

To demonstrate the effect of Jak2 activity on endothelial angiogenesis, we first used a Matrigel plug assay, with subcutaneous injection of Matrigel into Jak2-deficient and control mice. Significantly impaired endothelium-dependent neovascular formation was observed in Jak2-deficient mice, compared with that of control mice, as indicated by lower hemoglobin content (Figure 3, A and B) and fewer CD31 ${ }^{+}$ ECs (Figure 3C) in the gel plugs. To further confirm these findings, we used a hindlimb ischemia model. It is noteworthy that Jak2 expression underwent a time-dependent increase after ischemic surgery, and then gradually returned to a level similar to that under physiological conditions (Figure 4), suggesting that higher Jak2 activity is likely a prerequisite for prompt perfusion recovery. Indeed, a significant delay in restoration of blood flow was noted in Jak2-deficient mice after ischemic surgery (Figure 6). Importantly, unlike the Jak2 overactivation induced in diabetes, the increase of Jak2 activity in our ischemia model was transient, which further supports the hypothesis that transient increase of Jak2 activity is essential in enhancing endothelial angiogenesis for perfusion recovery but that sustained increase of Jak2 activity predisposes to endothelial dysfunction. As already noted, our focus in the present study was on demonstrating the immediate effect of Jak2 deficiency on the pathogenesis of endothelial dysfunction. We therefore did not use animal models with cardiovascular disorders such as hypertension, because the progression from endothelial dysfunction to development of hypertension is a chronic and complex process. Nonetheless, increased blood pressure and the resulting effect on blood flow could indirectly affect angiogenesis and reperfusion recovery after ischemic injury. Follow-up studies would be necessary to further address the influence of Jak2 deficiency on angiogenesis and reperfusion in hypertensive conditions.

To dissect the mechanisms by which loss of Jak2 impairs endothelial function, we performed antibody array analysis and identified a distinctive reduction in eNOS expression in Jak2-deficient mice. Western blot analysis revealed that both total and phosphorylated eNOS levels were significantly lower in Jak2-deficient mice than in littermate controls (Figure 6A). In contrast to a previous study in VSMCs, in which blockade of Jak2 by AG490 abolished angiotensin II-induced ERK1/2 activation, ${ }^{30}$ the present study unexpectedly demonstrated that loss of Jak2 did not result in any detectable change for either total or phosphorylated AKT (Figure 6B), ERK1/2 (Figure 6C), and p38 (data not shown), suggesting that Jak2 regulation of eNOS expression could be independent of AKT, ERK1/2, and p38 signaling. Again, this discrepant result could be caused by either the difference of cell type (VSMCs versus ECs) or the nonspecificity of Jak2 inhibitors.

Because Sp-1 acts as an essential transcription factor responsible for eNOS expression, we examined Sp-1 expression and its DNA binding activity. Western blot analysis of immunoprecipitates revealed that Jak2 deficiency significantly attenuated Sp-1 activation (Figure 7A), and inhibition of Jak2 activity with AZD1480 in PAECs consistently led to nearly a fourfold reduction in its DNA binding activity (Figure 7B). Given that loss of Jak2 did not suppress total Sp-1 protein levels (Figure 7A), we therefore hypothesized that loss of Jak 2 represses Sp-1 activity by attenuating downstream MAPK signaling. Western blot analysis of Jak2 downstream kinases confirmed that Jak2 deficiency significantly impaired the phosphorylation of tyrosines (at 340 and 341) in Raf-1 (Figure 6C). Phosphorylation of these two tyrosine residues has been suggested to be essential for Raf-1 activation. ${ }^{31}$ We then examined MEK1, a serine-threonine protein kinase downstream of Raf- $1 .^{20}$ As expected, a significant reduction in p-MEK1 was noted in Jak2-deficient mice, compared with that of control littermates (Figure 7D). Although previous studies in MCF7 breast cancer cells demonstrated that MEK1 directly regulates Sp-1 activity, ${ }^{32}$ whether MEK1 regulates Sp-1 activity in ECs is yet to be ascertained. We therefore treated PAECs with PD98059, a MEK1 inhibitor. Similar to the results in MCF7 cells, inhibition of MEK1 in PAECs attenuated TNF- $\alpha$-induced Sp-1 phosphorylation (Figure 7E) and reduced DNA binding activity of Sp-1 (Figure 7F). Taken together, these data provide evidence that loss of Jak2 reduces eNOS expression via impaired Raf-1/MEK1 signaling and attenuated Sp-1 transcriptional activity.

Given that ERK1 and ERK2 are well-recognized downstream molecules for Raf-1/MEK1 signaling, our mechanistic data are seemingly contradictory, in that reduced Raf-1/MEK1 activity did not result in a perceptible change in ERK $1 / 2$ activity in $J a k 2^{-/-}$mice. Of note, MEK1 has been recognized as a kinase with dual specificities that phosphorylates and activates ERK1/2 by phosphorylation on threonine and tyrosine, ${ }^{7}$ and one would therefore expect that reduced MEK1 activation would impair ERK1/2 activity. This discrepancy prompted us to recheck our original data by conducting additional experiments. The fresh results were consistent with the original data. We then checked the effect of the MEK1 inhibitor PD98059 on ERK1/2 activity in PAECs. Unlike the results in $\mathrm{Jak}^{-/-}$mice, treatment of PAECs with PD98059 resulted in a significant reduction in ERK1/2 activity (data not shown). Taken together, these conflicting results led us to consider that there might be an unidentified mechanism implicated in the regulation of ERK1/2 activity in the absence of Jak2. Indeed, several 
reports have consistently demonstrated the existence of RAF-independent ERK pathways. $^{1-4}$ Follow-up studies focused on dissecting the pathways implicated in the regulation of ERK1/2 activity in the absence of Jak2 would be important for fully understanding the pathogenic role of Jak2 in endothelial dysfunction.

In summary, we have presented evidence for loss of Jak2 leading to endothelial dysfunction, as indicated by altered vasodilator response capacity and impaired angiogenic capacity, along with delayed perfusion recovery after hindlimb ischemic surgery. In mechanistic studies, loss of Jak2 attenuated tyrosine phosphorylation of Raf-1, leading to reduced MEK1 activity, which then hampered Sp-1 activity and thereby impaired eNOS expression. The present findings not only provide novel information toward a greater understanding of the role of Jak2 in regulation of postnatal endothelial function, but might also help in assessing Jak2based therapeutic strategies against endothelial dysfunction in the setting of cardiovascular disorders.

\section{References}

1. Muñoz-Chápuli R, Quesada AR, Angel Medina M: Angiogenesis and signal transduction in endothelial cells. Cell Mol Life Sci 2004, 61: 2224-2243

2. Leibovich SJ, Polverini PJ, Shepard HM, Wiseman DM, Shively V, Nuseir N: Macrophage-induced angiogenesis is mediated by tumour necrosis factor-alpha. Nature 1987, 329:630-632

3. Sawhney R, Kabbinavar F: Angiogenesis and angiogenic inhibitors in renal cell carcinoma. Curr Urol Rep 2008, 9:26-33

4. Zetter BR: Cell motility in angiogenesis and tumor metastasis. Cancer Invest 1990, 8:669-671

5. Ichihara E, Kiura K, Tanimoto M: Targeting angiogenesis in cancer therapy. Acta Med Okayama 2011, 65:353-362

6. Mackey JR, Kerbel RS, Gelmon KA, McLeod DM, Chia SK, Rayson D, Verma S, Collins LL, Paterson AH, Robidoux A, Pritchard KI: Controlling angiogenesis in breast cancer: a systematic review of anti-angiogenic trials. Cancer Treat Rev 2012, 38:673-688

7. Shojaei F: Anti-angiogenesis therapy in cancer: current challenges and future perspectives. Cancer Lett 2012, 320:130-137

8. Schulz E, Gori T, Munzel T: Oxidative stress and endothelial dysfunction in hypertension. Hypertens Res 2011, 34:665-673

9. Hirase T, Node K: Endothelial dysfunction as a cellular mechanism for vascular failure. Am J Physiol Heart Circ Physiol 2012, 302: H499-H505

10. Balakumar P, Kathuria S: Submaximal PPARgamma activation and endothelial dysfunction: new perspectives for the management of cardiovascular disorders. Br J Pharmacol 2012, 166:1981-1992

11. Ghiadoni L, Taddei S, Virdis A: Hypertension and endothelial dysfunction: therapeutic approach. Curr Vasc Pharmacol 2012, 10: 42-60

12. Ihle JN, Gilliland DG: Jak2: normal function and role in hematopoietic disorders. Curr Opin Genet Dev 2007, 17:8-14

13. Sandberg EM, Wallace TA, Godeny MD, VonDerLinden D, Sayeski PP: Jak2 tyrosine kinase: a true jak of all trades? Cell Biochem Biophys 2004, 41:207-232

14. Zhong J, Yang P, Muta K, Dong R, Marrero M, Gong F, Wang CY: Loss of Jak2 selectively suppresses DC-mediated innate immune response and protects mice from lethal dose of LPS-induced septic shock. PLoS One 2010, 5:e9593
15. Watanabe S, Arai K: Roles of the JAK-STAT system in signal transduction via cytokine receptors. Curr Opin Genet Dev 1996, 6:587-596

16. Liu KD, Gaffen SL, Goldsmith MA: JAK/STAT signaling by cytokine receptors. Curr Opin Immunol 1998, 10:271-278

17. Banes-Berceli AK, Ketsawatsomkron P, Ogbi S, Patel B, Pollock DM, Marrero MB: Angiotensin II and endothelin-1 augment the vascular complications of diabetes via JAK2 activation. Am J Physiol Heart Circ Physiol 2007, 293:H1291-H1299

18. Levy DE, Darnell JE Jr: STATs: transcriptional control and biological impact. Nat Rev Mol Cell Biol 2002, 3:651-662

19. Guilluy C, Brégeon J, Toumaniantz G, Rolli-Derkinderen M, Retailleau K, Loufrani L, Henrion D, Scalbert E, Bril A, Torres RM, Offermanns S, Pacaud P, Loirand G: The Rho exchange factor Arhgef1 mediates the effects of angiotensin II on vascular tone and blood pressure. Nat Med 2010, 16:183-190

20. Krempler A, Qi Y, Triplett AA, Zhu J, Rui H, Wagner KU: Generation of a conditional knockout allele for the Janus kinase 2 (Jak2) gene in mice. Genesis 2004, 40:52-57

21. Ruzankina Y, Pinzon-Guzman C, Asare A, Ong T, Pontano L, Cotsarelis G, Zediak VP, Velez M, Bhandoola A, Brown EJ: Deletion of the developmentally essential gene ATR in adult mice leads to agerelated phenotypes and stem cell loss. Cell Stem Cell 2007, 1:113-126

22. Rao X, Zhong J, Zhang S, Zhang Y, Yu Q, Yang P, Wang MH, Fulton DJ, Shi H, Dong Z, Wang D, Wang CY: Loss of methyl-CpGbinding domain protein 2 enhances endothelial angiogenesis and protects mice against hind-limb ischemic injury. Circulation 2011, 123: 2964-2974

23. Han J, Zhong J, Wei W, Wang Y, Huang Y, Yang P, Purohit S, Dong Z, Wang MH, She JX, Gong F, Stern DM, Wang CY: Extracellular high-mobility group box 1 acts as an innate immune mediator to enhance autoimmune progression and diabetes onset in NOD mice. Diabetes 2008, 57:2118-2127

24. Kumar S, Sun X, Wiseman DA, Tian J, Umapathy NS, Verin AD, Black SM: Hydrogen peroxide decreases endothelial nitric oxide synthase promoter activity through the inhibition of Sp1 activity. DNA Cell Biol 2009, 28:119-129

25. Zhang R, Min W, Sessa WC: Functional analysis of the human endothelial nitric oxide synthase promoter. Sp1 and GATA factors are necessary for basal transcription in endothelial cells. J Biol Chem 1995, 270:15320-15326

26. Müller G, Storz P, Bourteele S, Döppler H, Pfizenmaier K, Mischak H, Philipp A, Kaiser C, Kolch W: Regulation of Raf-1 kinase by TNF via its second messenger ceramide and cross-talk with mitogenic signalling. EMBO J 1998, 17:732-742

27. Coles LC, Shaw PE: PAK1 primes MEK1 for phosphorylation by Raf1 kinase during cross-cascade activation of the ERK pathway. Oncogene 2002, 21:2236-2244

28. Reisinger K, Kaufmann R, Gille J: Increased Sp1 phosphorylation as a mechanism of hepatocyte growth factor (HGF/SF)-induced vascular endothelial growth factor (VEGF/VPF) transcription. J Cell Sci 2003, 116:225-238

29. Yamaoka K, Saharinen P, Pesu M, Holt VE 3rd, Silvennoinen O, O'Shea JJ: The Janus kinases (Jaks). Genome Biol 2004, 5:253

30. Marrero MB, Schieffer B, Li B, Sun J, Harp JB, Ling BN: Role of Janus kinase/signal transducer and activator of transcription and mitogen-activated protein kinase cascades in angiotensin II- and platelet-derived growth factor-induced vascular smooth muscle cell proliferation. J Biol Chem 1997, 272:24684-24690

31. Jelinek T, Dent P, Sturgill TW, Weber MJ: Ras-induced activation of Raf-1 is dependent on tyrosine phosphorylation, [Erratum appeared in Mol Cell Biol 1997, 17:2971]. Mol Cell Biol 1996, 16:1027-1034

32. Hockings JK, Degner SC, Morgan SS, Kemp MQ, Romagnolo DF: Involvement of a specificity proteins-binding element in regulation of basal and estrogen-induced transcription activity of the BRCA1 gene. Breast Cancer Res 2008, 10:R29 